Check for updates

Cite this: RSC Adv., 2017, 7, 39922

Received 9th June 2017

Accepted 25th July 2017

DOI: $10.1039 / c 7 r a 06463 b$

rsc.li/rsc-advances

\section{A low-power consumption MZI thermal optical switch with a graphene-assisted heating layer and air trench}

\author{
Yue Sun, Yue Cao, Yunji Yi, (D)* Liang Tian, Yao Zheng, Jie Zheng, Fei Wang \\ and Daming Zhang
}

To reduce the power consumption of thermal optical switches, graphene-assisted hybrid MZI structures were designed and simulated. The hybrid waveguide structures were composed of polymer cores, silica lower claddings, air trenches, and graphene-assisted heating layers. Because of the high thermal-optic coefficient of the polymer, excellent thermal conductivities of silica and graphene, and the air trench structures, the power consumption of the hybrid structures could be reduced to below $0.95 \mathrm{~mW}$. The graphene-assisted heating layers were also designed to bury the waveguide cores or to contact the surface of the waveguide cores for more efficiently conducting the heat of electrodes to the waveguide cores. Moreover, the side heating electrodes introduced were found to be compatible with the designed graphene-assisted heating layers. The polarization and the absorption of both rectangle and ridge waveguide structures were analyzed through optical field simulation. Based on the optimized parameters, the thermal fields were simulated, and the heating efficiencies of the graphene-assisted hybrid structures could be increased by $78 \%$ as compared to those of the top electrode device without a graphene layer. Our simulation comprises two MZI thermal optical switches with an optimized balance between the switching power consumption and the switching time. One MZI thermal optical switch with a lower power consumption realized the switching power consumptions of only $0.39 \mathrm{~mW}$ and the switching times of $30 \mu \mathrm{s}$ and $92.4 \mu \mathrm{s}$, whereas the other switch with faster switching times achieved the switching power consumptions of $0.95 \mathrm{~mW}$ and the switching times as fast as $1 \mu \mathrm{s}$ and $81.6 \mu \mathrm{s}$.

\section{Introduction}

Thermal-tuning optical devices include tunable filters, variable optical attenuators, and thermal optical switch. Thermal optical switch is a fundamental element in optical communication networks for realizing switching function through the thermooptic effect. The present research on a thermo-optic switch focuses on reducing the power consumption and improving the response speed from the aspects of material and structure.

From the aspects of material, polymer and inorganic material are two fundamental materials used in thermal optical switches. ${ }^{1-3}$ Polymer thermal optical switches have the advantage of low-power consumptions because of high thermal optical coefficient of the polymer. However, inorganic material thermal optical switches have a faster switching time. To achieve a balance between power consumption and switching time, hybrid integration with the polymer core and inorganic cladding has been massively investigated. In 2014, Liang Lei fabricated the organic/inorganic hybrid MZI thermo-optic switch with a switching power of $7.2 \mathrm{~mW}$ and response time of $100 \mu \mathrm{s}{ }^{4}$

State Key Laboratory on Integrated Optoelectronics, College of Electronic Science \& Engineering, Jilin University, Changchun 130012, P. R. China.E-mail: yiyj@jlu.edu.cn
In 2014, Xie Nan fabricated low-power, high-speed polymer buried channel waveguide thermo-optic switches with a switching power of $3.5 \mathrm{~mW}$ and a response time of $200 \mu \mathrm{s} .^{5}$ The abovementioned hybrid devices take the materials into account, but the structures of cladding and substrate have not been optimized.

From the aspects of structure, addition of air trenches to thermal optical switches is an effective way to reduce the power consumption. With a cladding air trench, the thermal field was mainly restricted in the waveguide region. In 2008, a novel $2 \times 2$ thermo-optic MMI switch was designed, where only one heat electrode was used with air and silicon trenches, and the applied power was $1.35 \mathrm{~mW} .^{6}$ In 2015 , a thermal optical switch with an air trench (switching time of $150 \mu$ s and the power consumption of $3.4 \mathrm{~mW}$ ) has been reported. ${ }^{7}$ With a substrate air trench, the mutual interference of the tuning branch and untuning branch could be eliminated. Even for the trench structure, a large portion of the heating power was wasted by the waveguide cladding. The function of the cladding was to reduce the optical loss caused by the metal electrode absorption. Thus, the electrode absorption would be a main restriction for the further reduction of the power consumption. A low optical absorption electrode material fabricated beside or in contact 
with the optical waveguide core would be a feasible solution to this problem.

Graphene, as a revolutionary two-dimensional (2D) sheet material with extraordinary mechanical, electronic, and photonic properties, has attracted significant attention in many fields. In the field of optics, graphene has been integrated with a waveguide device used for the polarizer ${ }^{8}$ detector, ${ }^{9}$ modulator, ${ }^{10,11}$ and optical switch. ${ }^{12}$ For the thermal optical switches, the integration of graphene and polymer will also lead to a revolutionary promotion of the switching properties. In 2014, non-local heating for thermally tuning nano-photonic has been achieved using a graphene heat conductor, and the $90 \%$ rising and decaying times are simulated to be $9 \mu \mathrm{s} .{ }^{13}$ In 2015 , graphene has been introduced as a transparent nanoheater of the thermally photonic microdisk resonators, and the rising time and decaying time are measured to be $12.8 \mu \mathrm{s}$ and $8.8 \mu \mathrm{s}$, respectively. ${ }^{14}$ Graphene-based waveguide thermal-optic switch is an effective way to increase the heating efficiency. Graphene has been used as a heat conductor or heating electrode of silicon waveguide due to its feature of splendid thermal conductivity and electro-conductivity. ${ }^{15,16}$

In this study, we have demonstrated a graphene-based hybrid waveguide thermal optical switch with an air trench. Based on the polymer core, inorganic lower cladding, and air trench, a graphene-assisted heating layer was designed to bury or contact the polymer waveguide core. Moreover, two waveguide structures and two graphene locations were designed. In addition, the polarization, absorption, heating efficiency, and switching properties were simulated and optimized.

\section{Simulation and design}

\section{Device structure}

The device structure was composed of a polymer waveguide core, metal electrode, and graphene-assisted heating layer. The metal electrode was located beside the waveguide, ${ }^{17}$ and the

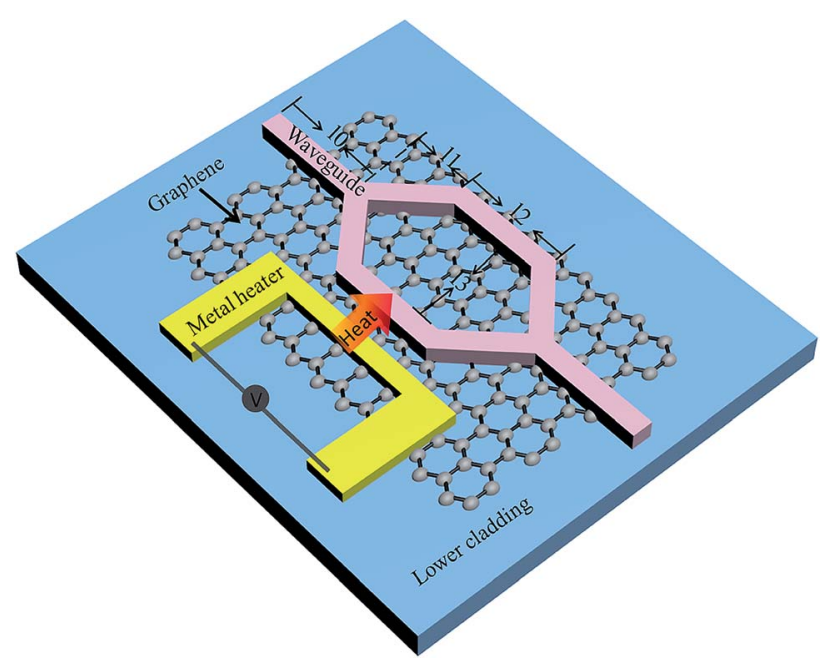

Fig. 1 The structure of the thermal optical switch with a grapheneassisted heating layer. graphene-assisted heating layer was contacted or buried in the waveguide core, as was shown in Fig. 1.

The position of graphene determines the optical loss and the thermal filed distribution. Based on the position of graphene (in the centre or at the bottom) and the waveguide type (rectangular or ridge structure), four basic structures were simulated, as shown in Fig. 2. The corresponding parameters are indicated in the figure, and the dimension parameters of the waveguide are $w$ (width), $h$ (height), $h_{1}$ (ridge height), and $d$ (separation width between the waveguide and the side metal heating electrode). SU-8 (refractive index: 1.575@1550 nm) was selected as the polymer waveguide core material due to its lithography and photobleaching fabrication methods, which were compatible with the waveguide structure and graphene transfer method. Due to different material features, silicon was selected as the substrate and heat sink with the thickness of $5 \mu \mathrm{m}$. As the lower cladding material, silica (refractive index: 1.450) was compared with the PMMA (refractive index: 1.490) material. Air and PMMA have been selected as the upper cladding, and the refractive index is 1 and 1.490, respectively. The heater material is gold, and its refractive index is $0.559+11.5 \mathrm{i}$. The refractive index of graphene is $2.973+2.79 \mathrm{i} .{ }^{18}$

\section{Optical field}

To ensure the single-mode condition, the rectangle waveguide dimension was selected to be $2 \mu \mathrm{m} \times 2 \mu \mathrm{m}$, and the ridge waveguide width $(w)$, ridge height $\left(h_{1}\right)$, and waveguide height $(h)$ were selected to be $3 \mu \mathrm{m}, 3 \mu \mathrm{m}$, and $5 \mu \mathrm{m}$, respectively. The integrated graphene layer would bring polarization absorption to the waveguide, and the absorption of TE and TM mode caused by the graphene layer was simulated. ${ }^{19,20}$ Fig. 3 shows the effective optical field distribution of the TE and TM mode. When the graphene layer was at the bottom of the core, the effective optical field of the TE-pass polarizer was simulated, as shown in (a) and (c). Moreover, the effective optical field of the TM-pass polarizer was simulated, as shown in (b) and (d), with
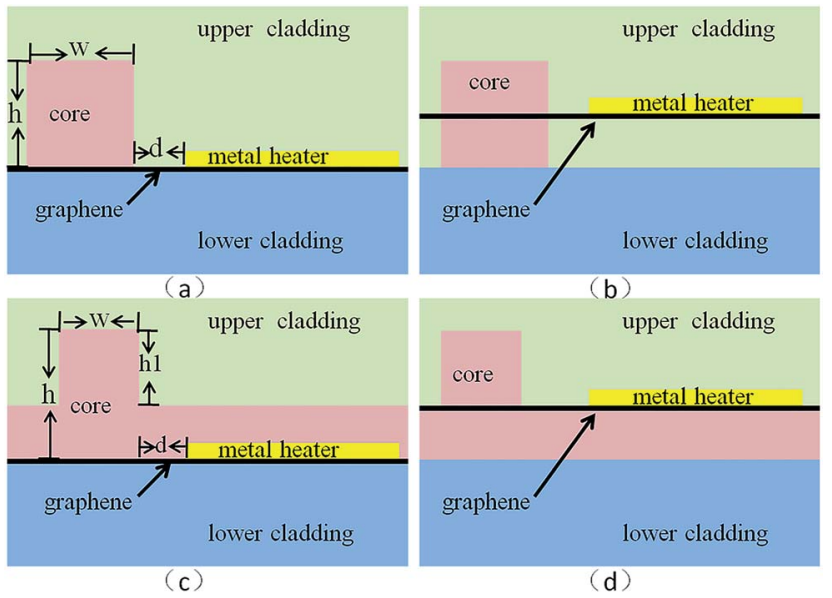

Fig. 2 The four structures of a thermal optical switch with a grapheneassisted heating layer: (a) bottom heating rectangular structure, (b) centre heating rectangular structure, (c) bottom heating ridge structure, and (d) centre heating ridge structure. 


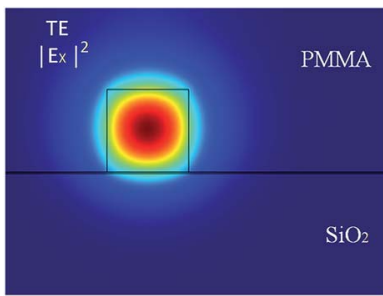

(a)

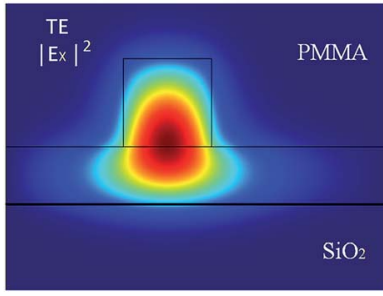

(c)

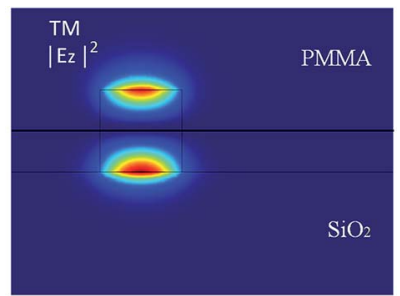

(b)

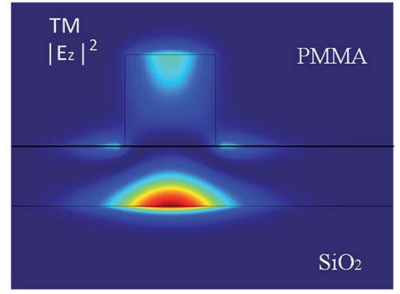

(d)
Fig. 3 The effective optical field for graphene absorption. (a) The effective optical field of the TE mode in the rectangle waveguide. (b) The effective optical field of the TM mode in the rectangle waveguide. (c) The effective optical field of the TE mode in the ridge waveguide. (d) The effective optical field of the TM mode in the ridge waveguide.

the graphene layer at the centre of the core. The optical absorption of the $1 \mathrm{~cm}$ graphene conductive layer of the TE and TM mode is calculated to be $94.36 \mathrm{~dB}$ and $13.86 \mathrm{~dB}$ of the bottom heater rectangular waveguide structure, respectively. For the centre heater rectangular waveguide structure, the optical absorption of the TE and TM mode is calculated to be $480.84 \mathrm{~dB}$ and $10.5 \mathrm{~dB}$, respectively. For the bottom heater ridge waveguide structure, the optical absorption for the TE and TM mode is calculated to be $28.68 \mathrm{~dB}$ and $4.24 \mathrm{~dB}$, respectively. The optical absorption of the TE and TM mode is calculated to be $244.74 \mathrm{~dB}$ and $6.59 \mathrm{~dB}$ for the center heater ridge waveguide structure, respectively. The equation is as follows:

$$
\text { Absorption }=10 L k N_{\text {neff }}=10 L 2 \pi / \lambda N_{\text {neff }}
$$

where $L$ is the length of graphene, $\lambda$ is the wavelength, and $N_{\text {neff }}$ is the effective refractive index.

Due to the absorption of the electrode, the distance between the waveguide and the electrode should be optimized to ensure a high heating efficiency and low optical loss. The absorption of rectangular and ridge waveguide with the changing distance between the waveguide and the electrode is shown in Fig. 4. For the rectangular waveguide structure, when the electrodes were located at the bottom of the core, the electrode had only a little influence on the optical absorption. Moreover, when the electrode was located at the side of the rectangle waveguide core, the distance between the rectangle waveguide and the electrode was optimized to be larger than $1 \mu \mathrm{m}$. In the ridge waveguide structure, the optimized distance between the waveguide and the electrode was $3 \mu \mathrm{m}$, as shown in Fig. 4(b).

\section{Thermal field optimization}

The thermal field is associated with the heating efficiency of the electrode. When the temperature contrast is fixed, a higher

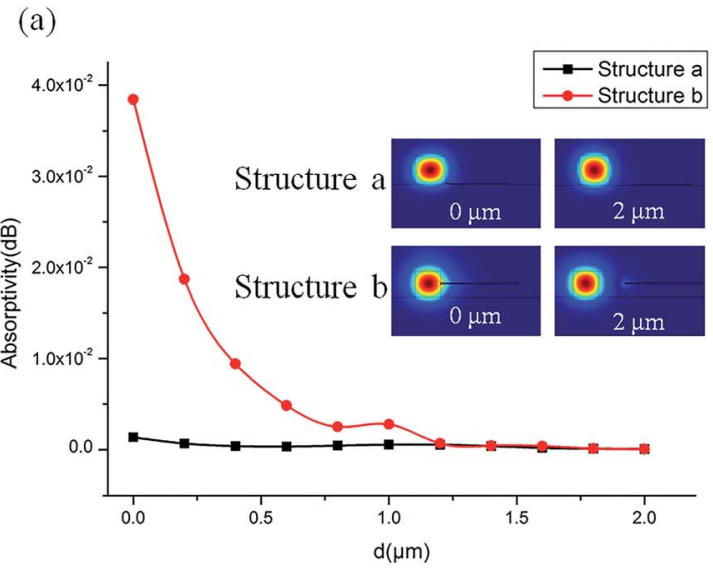

(b)

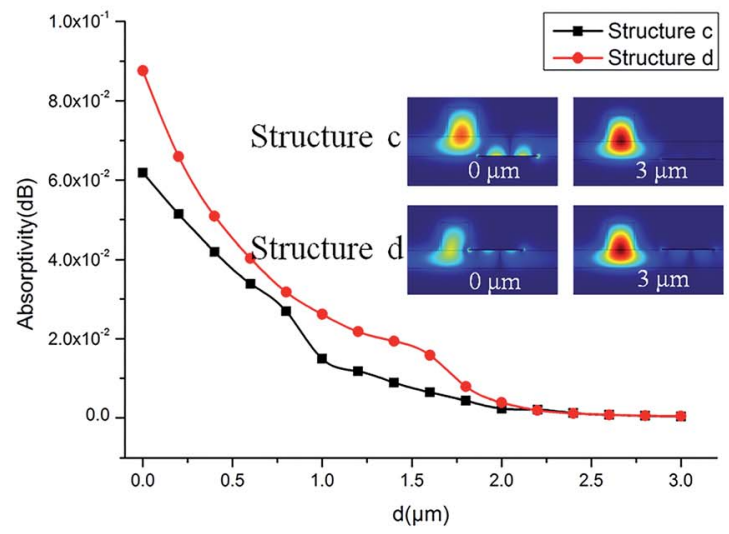

Fig. 4 (a) The absorption of the gold electrode of rectangular waveguide structure $1550 \mathrm{~nm}$. (b) The absorption of the gold electrode of ridge waveguide structure $(1550 \mathrm{~nm}$.

waveguide core temperature means a higher heating efficiency. The relationship between the heating efficiency and the distance between the waveguide core and the electrode was simulated, the simulation parameters are listed below: SU-8 (thermal conductivity: $0.2 \mathrm{~W} \mathrm{~m}^{-1} \mathrm{~K}^{-1}$ ), silica (thermal conductivity: $0.27 \mathrm{~W} \mathrm{~m}^{-1} \mathrm{~K}^{-1}$ ), PMMA (thermal conductivity: $0.19 \mathrm{~W} \mathrm{~m}^{-1} \mathrm{~K}^{-1}$ ), air (thermal conductivity: $0.023 \mathrm{~W} \mathrm{~m}^{-1} \mathrm{~K}^{-1}$ ), and graphene (thermal conductivity: $5300 \mathrm{~W} \mathrm{~m}^{-1} \mathrm{~K}^{-1}$ ). The gold heater is $0.1 \mu \mathrm{m}$ thick and has a width of $6 \mu \mathrm{m}$. The external temperature is $295 \mathrm{~K}$. The relation between the temperature of the core centre and the distance between electrode and core is shown in Fig. 5. The part of the steady-state thermal field is shown in the inset of Fig. 5. The effect of distance between the heater and the core layer on the heating efficiency is calculated and compared.

Compared to that of the polymer, the thermal conductivity of air is $1 / 10$ to polymer. The comparison of the thermal field between the structure with polymer upper cladding and the structure with air upper cladding is shown in Fig. 6. In the rectangular structure (Fig. 6(a) and (b)), which had a metal heater next to the core, the central temperature of the waveguide was $297.42 \mathrm{~K}$ and $298.36 \mathrm{~K}$, respectively. With air cladding, the thermal field can be concentrated in the waveguide core layer, and the heating efficiency is superior. 


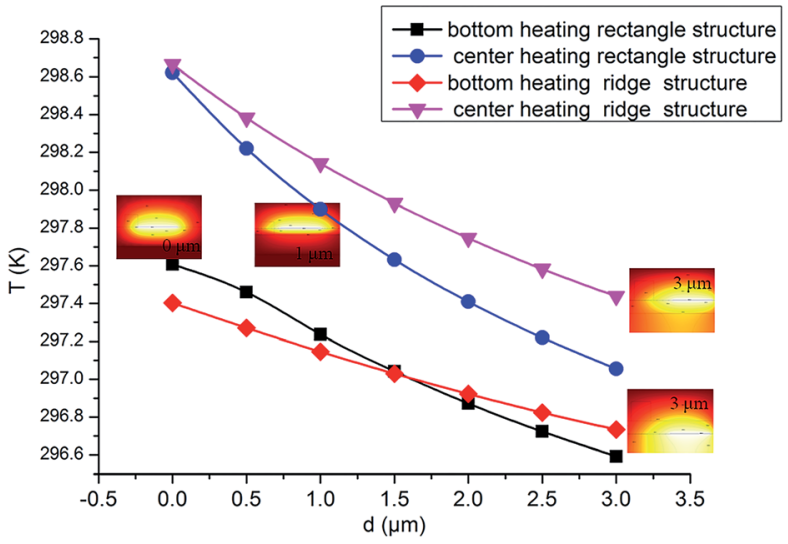

Fig. 5 The relation between the central temperature of core $(T)$ and the width between waveguide and side electrode $(d)$.
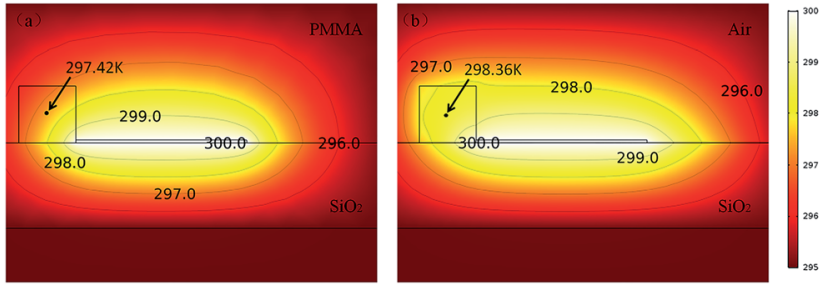

Fig. 6 The thermal field (a) polymer upper cladding and (b) air upper cladding.

Compared with that of silica, the thermal conductivity of the polymer is relatively poor. The thermal field of the polymer lower cladding structure was simulated and compared with that of the silica lower cladding structure, as shown in Fig. 7. It can be seen that the influence of the lower cladding material is not obvious. The deep air trench structure has been ameliorated (see Fig. 8(a)), and the temperature of the waveguide core (299.44 K) was greatly improved.

One of the unique features of polymer materials is their flexibility. By taking advantage of their flexibility, we designed the structure shown in Fig. 8(b). Moreover, the thin layer of the polymer is doped with a highly thermally conductive material as the lower cladding of the waveguide. The flexible waveguide with a high thermal conductivity can thus be implemented (thermal conductivity: $500 \mathrm{~W} \mathrm{~m}^{-1} \mathrm{~K}^{-1}$ ).
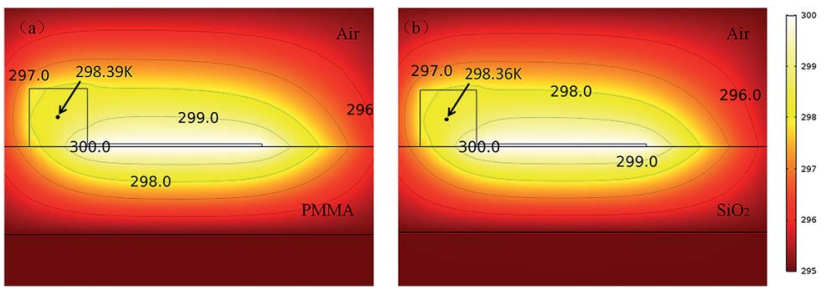

Fig. 7 The thermal field (a) polymer lower cladding and (b) inorganic lower cladding.

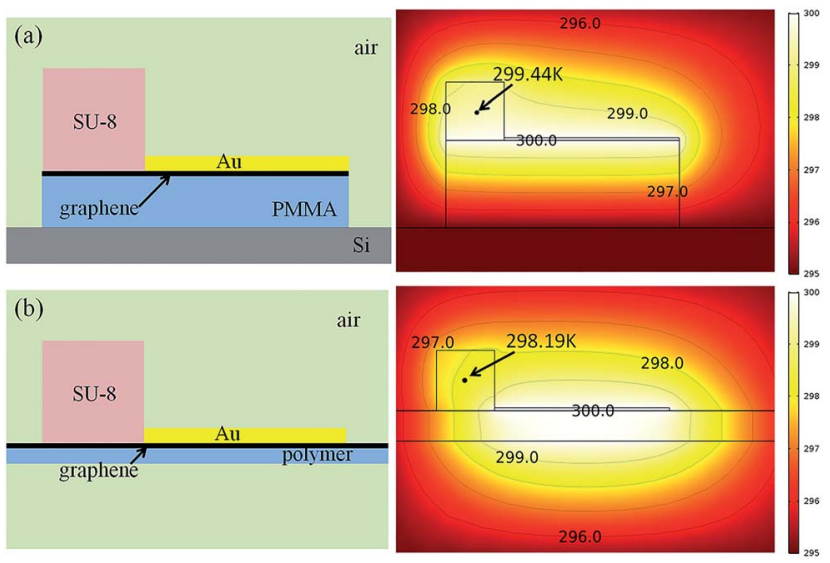

Fig. 8 The structure and thermal field (a) ameliorated air trench structure and (b) flexible structure.

To balance the optical absorption and the heating efficiency, the optimized parameters of the four structures are shown in Table 1.

To compare the power consumption of the top electrode polymer thermal optical switch and the graphene-assisted ones, the heating electrode length $\left(l_{2}\right)$ was selected to be equal to the

Table 1 The optimized parameters of four structures

\begin{tabular}{lllll}
\hline Length $(\mu \mathrm{m})$ date structure & $w$ & $h$ & $h_{1}$ & $d$ \\
\hline Bottom heating rectangular structure & 2 & 2 & - & 0 \\
Centre heating rectangular structure & 2 & 2 & - & 1 \\
Bottom heating ridge structure & 3 & 5 & 3 & 3 \\
Centre heating ridge structure & 3 & 5 & 3 & 3
\end{tabular}
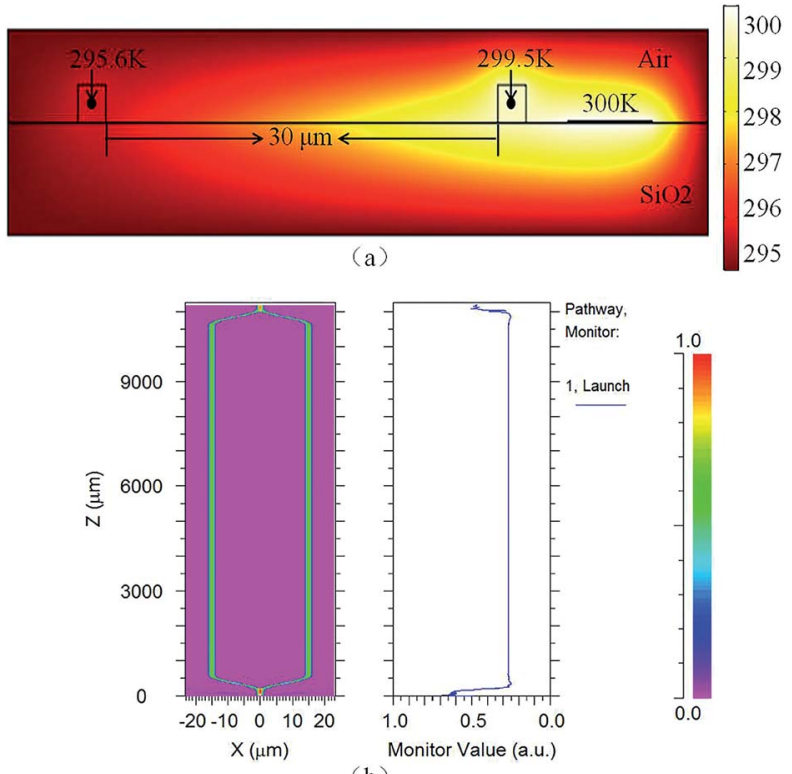

(b)

Fig. 9 (a) The thermal field and (b) optical field of the switch when $l_{1}=$ $500 \mu \mathrm{m}, l_{2}=1 \mathrm{~cm}$, and $l_{3}=30 \mu \mathrm{m}$. 
length of the $\mathrm{M}-\mathrm{Z}$ branch, which had a length of $1 \mathrm{~cm}$. The distance between the two arms of the M-Z structure was selected to be $30 \mu \mathrm{m}\left(l_{3}\right)$. As is shown in Fig. 9(a), the heating electrode

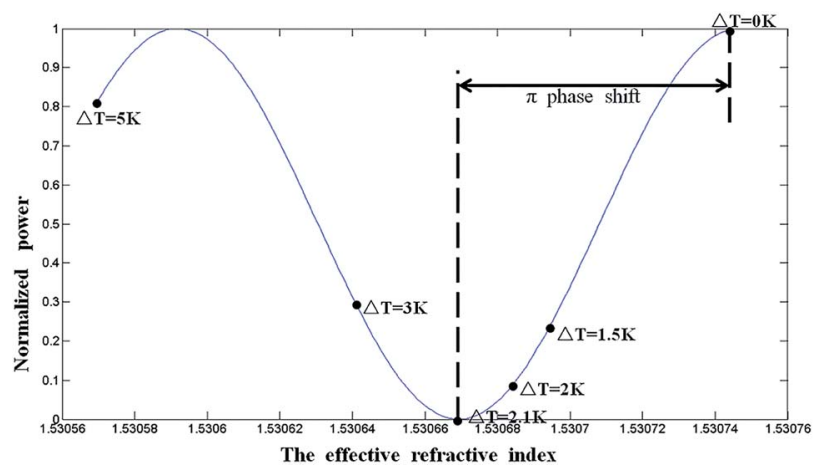

Fig. 10 The relation between the phase change and the temperature change of the heater.
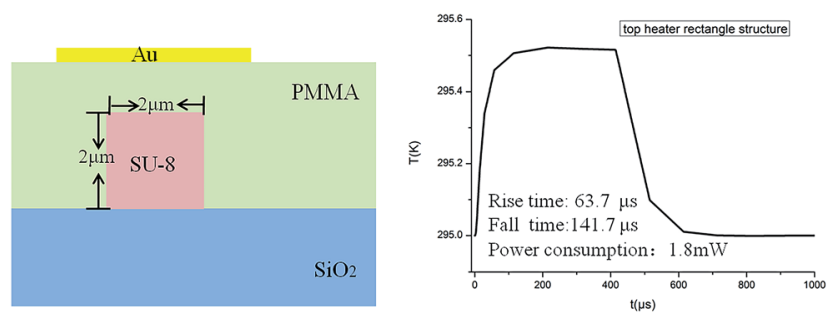

Fig. 11 Cross-section of conventional waveguide with top heater and the relationship between temperature and time.
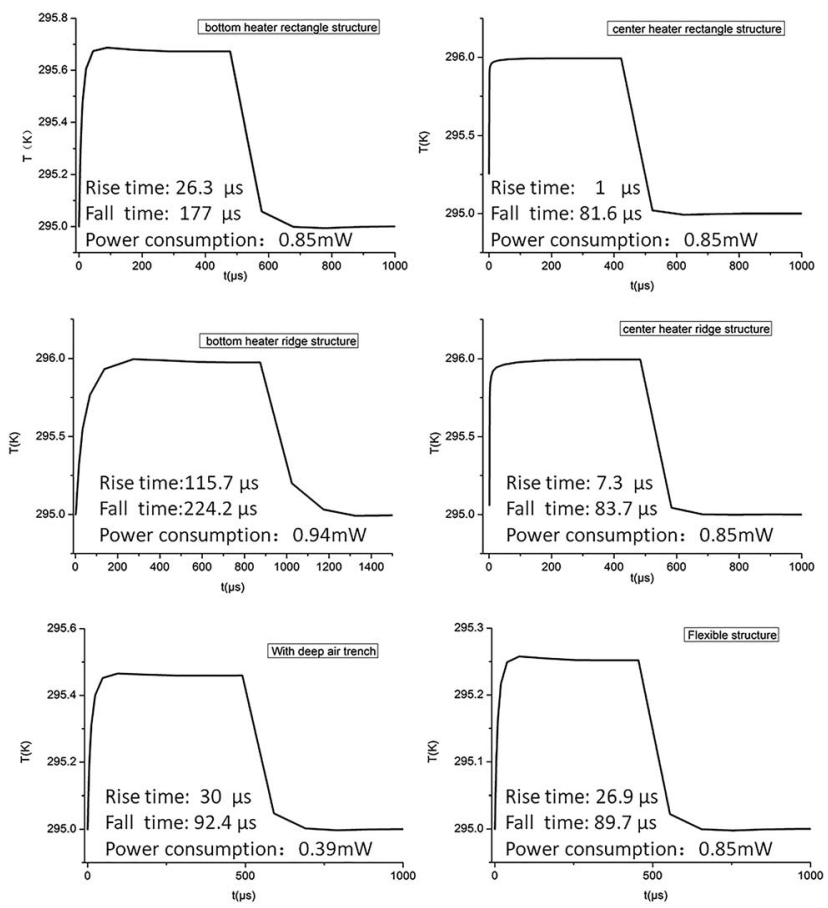

Fig. 12 The relationship between temperature and time of different structures and corresponding rise time, fall time, and power consumption. has little influence on the other waveguide. The corresponding parameters of $l_{0}$ and $l_{1}$ were $500 \mu \mathrm{m}$ and $500 \mu \mathrm{m}$, respectively. The optical field of the device is shown in Fig. 9(b).

According to the interference theory of the thermo-optical switch, the phase shift is associated with the effective index changes caused by the electrode. The change of effective refractive index was simulated with the changing electrode heating temperature. When the phase change is $1 / 4$ cycles, the thermo optic switch has a switching effect. Fig. 10 shows the relation between the phase changes and the temperature changes of the heater (the device structure as shown in Fig. 11, the conventional waveguide with top heater). Herein, $2.1 \mathrm{~K}$ is the required temperature. The rise time, fall time, and power consumption were calculated, as shown in the right of Fig. 11.

According to the analysis of the light and thermal field of the four kinds of structures, the switching rise time and fall time were calculated (see the Fig. 12).

\section{Conclusions}

Hybrid MZI thermal optical switches with a graphene-assisted heating layer and air trench and a power consumption of below $0.95 \mathrm{~mW}$ were proposed. Due to the air trench and excellent thermal conductivity of graphene, the heating efficiency can be increased by $45-78 \%$ as compared to that of the traditional device. The optimized central heating rectangle structure possess the rise and fall time of $1 \mu \mathrm{s}$ and $81.6 \mu \mathrm{s}$, respectively, with a power consumption of $0.85 \mathrm{~mW}$. The optimized deep air trenching central heating rectangle structure possess the rise and fall time of $30 \mu$ s and $92.4 \mu$ s, respectively, with the power consumption of $0.39 \mathrm{~mW}$.

\section{Acknowledgements}

This work was supported by the National Natural Science Foundation of China (NSFC) [61605057, 61475061, 61575076; 61377058], Science and Technology Development Plan of Jilin Province [20140519006JH], and Excellent Youth Foundation of Jilin Province [20170520158JH].

\section{Notes and references}

1 R. L. Espinola, M.-C. Tsai, J. T. Yardley and R. M. Osgood Jr, Fast and low-power thermo optic switch on thin silicon-oninsulator, IEEE Photonics Technol. Lett., 2003, 15(10), 13661368.

2 L. Liang, C.-T. Zheng, Y.-F. Yan, X.-Q. Sun, F. Wang, C. S. Ma and D.-M. Zhang, Low-power and high-speed thermo-optic switch using hybrid silica/polymer waveguide structure: design, fabrication and measurement, J. Mod. Opt., 2012, 59(12), 1084-1091.

3 Y.-F. Liu, X.-B. Wang, J.-W. Sun, J. Sun, F. Wang, C.-M. Chen, X.-Q. Sun, Z.-C. Cui and D.-M. Zhang, Improved performance of thermal-optic switch using polymer/silica hybrid and air trench waveguide structures, Opt. Lett., 2015, 40(9), 18881891. 
4 L. Liang, L. Qv, L. Zhang, C. Zheng, X. Sun, F. Wang and D. Zhang, Fabrication and characterization on an organic/ inorganic $2 \times 2$ Mach-Zehnder interferometer thermo-optic switch, Photonics and Nanostructures, 2014, 12(2), 173-183.

$5 \mathrm{X}$. Nan, H. Takafumi and U. Katsuyuki, Design and Performance of Low-Power, High-Speed, PolarizationIndependent and Wideband Polymer Buried Channel Waveguide Thermo-Optic Switches, J. Lightwave Technol., 2014, 32(17), 3067-3073.

6 A. M. Al-Hetar, I. Yulianti, A. S. M. Supa'At, et al., Thermooptic multimode interference switches with air and silicon trenches, Opt. Commun., 2008, 281(18), 4653-4657.

7 Y.-F. Liu, X.-B. Wang, J. Sun, H.-J. Gu, X.-Q. Sun, C.-M. Chen, F. Wang and D.-M. Zhang, Thermal field analysis of polymer/ silica hybrid waveguide thermo-optic switch, Opt. Commun., 2015, 356, 79-83.

8 X. Yin, T. Zhang, L. Chen and X. Li, Ultra-compact TE-pass polarizer with graphene multilayer embedded in a silicon slot waveguide, Opt. Lett., 2015, 40(8), 1733-1736.

9 L. Yu, J. Zheng, Y. Xu, D. Dai and S. He, Local and nonlocal optically induced transparency effects in graphene silicon hybrid nanophotonic integrated circuits, ACS Nano, 2014, 8(11), 11386-11393.

10 S. Gan, C. Cheng, Y. Zhan, B. Huang, X. Gan, S. Li, S. Lin, X. Li, J. Zhao, H. Chen and Q. Bao, A highly efficient thermo-optic microrin modulator assisted by graphene, Nanoscale, 2015, 7, 20249-20255.
11 M. Liu, X. Yin, E. Ulin-Avila, B. Geng, T. Zentgraf, L. Ju, F. Wang and $\mathrm{X}$. Zhang, A graphene-based broadband optical modulator, Nature, 2011, 474, 64-67.

12 X. Gan, C. Zhao, Y. Wang, D. Mao, L. Fang, L. Han and J. Zhao, Graphene-assisted all-fiber phase shifter and switching, Optica, 2015, 2(5), 468-471.

13 L. Yu, Y. Yin, Y. Shi, D. Dai and S. He, Thermally tunable silicon photonic microdisk resonator with transparent graphene nanoheaters, Optica, 2016, 3(2), 159-166.

14 L. Yu, D. Dai and S. He, Graphene-based transparent flexible heat conductor for thermally tuning nanophotonic integrated devices, Appl. Phys. Lett., 2014, 105, 251104.

15 K. S. Novoselov, A. K. Geim, S. V. Morozov, D. Jiang, Y. Zhang, S. V. Dubonos, I. V. Grigorieva and A. A. Firsov, Electric field effect in atomically thin carbon films, Science, 2004, 306(5696), 666.

16 R. Prasher, Graphene spreads the heat, Science, 2010, 328, 185-186.

17 Z. Chang and K. S. Chiang, Experimental verification of optical models of graphene with multimode slab waveguides, Opt. Lett., 2016, 41(9), 2129-2132.

18 Z. Zhang and N. Keil, Thermo-optic devices on polymer platform, Opt. Commun., 2016, 362, 101-114.

19 Q. Bao and K. Ping Loh, Graphene photonics, plasmonics, and broadband optoelectronic devices, ACS Nano, 2012, 6(5), 3677-3694.

20 J. W. Weber, V. E. Calado and M. C. M. van de Sanden, Optical constants of graphene measured by spectroscopic ellipsometry, Appl. Phys. Lett., 2010, 97, 091904. 